\title{
Review \\ VEGF and Delta-Notch: interacting signalling pathways in tumour angiogenesis
}

\author{
G Thurston*,1 and J Kitajewski*,2 \\ 'Regeneron Pharmaceuticals, 777 Old Saw Mill River Road, Tarrytown, New York, NY I059I, USA; ${ }^{2}$ Herbert Irving Comprehensive Cancer Center, \\ Columbia University, I 130 St Nicholas Avenue, New York, NY 10032, USA
}

Tumour angiogenesis has become an important target for antitumour therapy, with most current therapies aimed at blocking the VEGF pathway. However, not all tumours are responsive to VEGF blockers, and some tumours that are responsive initially may become resistant during the course of treatment, thus there is a need to explore other angiogenesis signalling pathways. Recently, the Delta-Notch pathway, and particularly the ligand Delta-like 4 (D\|l4), was identified as a new target in tumour angiogenesis. An important feature in angiogenesis is the manifold ways in which the VEGF and Delta-Notch pathways interact. The emerging picture is that the VEGF pathway acts as a potent upstream activating stimulus for angiogenesis, whereas Delta-Notch helps to guide cell fate decisions that appropriately shape the activation. Here we review the two signalling pathways and what is currently known about the ways in which they interact during tumour angiogenesis.

British Journal of Cancer (2008) 99, 1204-1209. doi:I0.1038/sj.bjc.6604484 www.bjcancer.com

Published online 30 September 2008

(c) 2008 Cancer Research UK

Keywords: DII4; endothelial; Notch I; Kdr; anti-angiogenesis

Solid tumours require the growth of new blood vessels (angiogenesis) to grow (Folkman, 2002). Tumour angiogenesis utilises at least some of the angiogenic signalling pathways that are required during vascular development. Over the past 10 years, these pathways have been recognised as important targets for antitumour therapy, and a number of different approaches to block angiogenic signalling pathways, and thus block tumour growth, have been developed.

At the present time, blocking the VEGF pathway is the bestvalidated approach to blocking tumour angiogenesis. Studies from a number of years ago showed that the VEGF pathway is central and absolutely essential for early stages of developmental angiogenesis; these findings helped drive the development of VEGF inhibitors for clinical use (Ferrara, 1999). However, unlike developmental angiogenesis, not all tumours are responsive to VEGF blockers, and some tumours that are responsive initially may become resistant during the course of treatment. Thus, there is a need to explore other angiogenesis signalling pathways as therapeutic targets, including those that interact with the VEGF pathway.

One such pathway is Delta/Jagged-Notch signalling system, which is generally involved in cell fate decisions. One particular ligand, Dll4, was recently identified as a potentially important new target in tumour angiogenesis. Other members of this pathway, including Notch receptors, are also required for developmental angiogenesis. The emerging picture is that the VEGF pathway acts as a potent upstream activating stimulus for angiogenesis, whereas Delta-Notch helps to shape that activation appropriately. An

*Correspondence: Dr G Thurston; E-mail: Gavin.Thurston@regeneron.com or J Kitajewski; E-mail: jkk9@columbia.edu

Received 20 December 2007; revised 5 June 2008; accepted 6 June 2008; published online 30 September 2008 important feature of angiogenesis is the manifold ways in which the VEGF and Delta-Notch pathways interact.

In this review, we briefly summarise the components and features of the VEGF and Delta-Notch pathways, including their roles in developmental and tumour angiogenesis. Then we summarise in more detail what is currently known about the ways in which these pathways interact in the vasculature. A deeper understanding of these interactions should lead to better use of agents that block the Dll4-Notch pathway, alone and in combination with currently used inhibitors of the VEGF pathway.

\section{THE VEGF PATHWAY}

\section{Molecular components, general features and functions}

The VEGF system has been conserved from zebrafish to man as a signalling pathway that is essential and rather specific to the vascular and haematopoietic systems. In mammals, the system comprises five secreted ligands (VEGF-A, VEGF-B, VEGF-C, VEGF-D and PlGF) and three primary receptors (VEGF-R1, VEGF-R2, VEGF-R3). In general, the ligands are expressed rather broadly and display multiple splice isoforms of varying size and degree of interaction with the extracellular matrix. An important point is that the expression of VEGF-A is induced by hypoxia, which is a common feature of rapidly growing solid tumours, and constitutes a key signal from a tissue calling for more blood vessels and/or increased vascular function. In contrast to the ligands, the receptors show much more restricted cell-type expression. VEGF$\mathrm{R} 1$ and VEGF-R2 are prominently expressed by vascular endothelial cells in addition to expression by selected other cell types. VEGF-R3 is prominently expressed in lymphatic endothelial cells, although it can also be expressed on activated blood endothelial cells (Tammela et al, 2005). The ligands show discrete binding to 
specific receptor(s); for example, VEGF-A interacts with VEGF-R1 and VEGF-R2, whereas PlGF interacts strictly with VEGF-R1. Additional components of the system include the cell-surface proteins, neuropilin-1 and -2 , which can serve as coreceptors on endothelial cells for larger isoforms of VEGF-A and VEGF-C. The VEGF receptors are tyrosine kinases that autophosphorylate upon dimerisation by ligand, which in turn activates various downstream signalling pathways. Signals from VEGF receptors drive diverse cellular effects in endothelial cells, including migration, proliferation, cell survival and the expression of downstream genes.

\section{Genetic deletion in mice and knockdown in zebrafish}

Genetic targeting studies in mice have emphasised the essential nature of the VEGF pathway for the development of the blood and lymphatic vascular systems. Genetic deletion of $V E G F-A$ or its primary signalling receptor $V E G F-R 2$ results in early embryonic lethality (approximately embryonic day E9), associated with a near-complete block of haematopoietic and vascular development. Somewhat analogously, deletion of $V E G F-C$ results in a severe block in the development of the lymphatic system. Deletion of the main receptor for VEGF-C, VEGF-R3, results in embryonic lethality associated with blood vascular defects; however, this receptor becomes essential for the development of the lymphatic system slightly later in development (Tammela et al, 2005). Deletion of VEGF-R1 also results in embryonic lethality, although in this case, the defect is an overabundance of blood vessels. Deletion of other VEGF ligands ( $P l G F, V E G F-B, V E G F-D)$ is not lethal and is associated with much more subtle phenotypes. Studies in zebrafish also reveal essential upstream roles for the VEGF system: high-dose morpholino knockdown of VEGF-A in early zebrafish embryos (day 2) results in a near-total absence of vasculature (similar to results in mice), whereas lower dose VEGF-A knockdown results in severe disruption of the dorsal aorta and intersegmental arteries (Nasevicius et al, 2000; Lawson et al, 2002).

\section{Blockade of the VEGF pathway in tumours}

The VEGF pathway was identified as a target for antitumour therapy more than 15 years ago, and early studies showed that blocking the VEGF-A ligand with a monoclonal antibody could result in strong suppression of tumour growth (Ferrara, 1999). Since these initial studies, many different approaches have been taken to block the VEGF pathways, focusing on VEGF-A ligand and VEGF-R2 receptor (Duda et al, 2007). Various preclinical studies have shown that successful blockade of the pathway in tumourbearing mice results in vessel pruning and reduction of angiogenic sprouting, which produce a 'normalisation' of the aberrant tumour vessels (Duda et al, 2007). The reduced tumour angiogenesis leads to reduced tumour growth in a broad variety of tumour types. These preclinical results have been validated by successful phase 3 clinical trials of anti-VEGF agents in several types of cancers, including colorectal, renal cell carcinoma and breast cancer. Studies in numerous cancer indications are currently ongoing.

\section{THE DELTA/JAGGED-NOTCH PATHWAY}

\section{Molecular components, general features and functions}

Notch is a fundamental signalling pathway in many mammalian cell types undergoing differentiation, primarily acting to regulate cell fate determination. Delta-Notch signalling is a form of cellcell communication, helping a group of similar cells to integrate contextual information and steer down separate pathways of differentiation. One mechanism, referred to as lateral inhibition, involves presentation of a Notch ligand (Delta or Jagged) to an adjacent Notch receptor cell, resulting in activation of the Notch pathway in one cell and suppression in the adjacent cells. The end result locks the cells into distinct cellular fates; one promoted by Notch signal activation and the other dependent on lack of Notch signalling. Notch receptors are distinct in that they operate both on the cell surface to bind ligand and within the nucleus as transcriptional modulators (Mumm and Kopan, 2000). The pathway in mammals is comprised of a conserved family of four transmembrane receptors (Notch1-4). In the vasculature, Notch1 and Notch4 function in developing endothelium, whereas Notch3 is critical for smooth muscle cell differentiation. Mammals utilise five membrane-bound Notch ligands (Delta-like 1, 3, 4, Jagged1 and -2). Those ligands implicated in the process of angiogenesis include Delta-like 1 (Dll1), Delta-like 4 (Dll4) and Jagged1. Receptor-ligand interactions between adjacent cells first trigger the metalloproteinase ADAM (a disintegrin and metalloproteinase) to cleave the Notch extracellular region and then trigger $\gamma$-secretase-mediated generation of the Notch intracellular domain (NICD). Subsequently, NICD translocates to the nucleus, where it interacts with the CSL (RBPJ) transcription factor to induce multiple downstream targets, including a family of basic helixloop-helix proteins, the Hes and Hey genes (Mumm and Kopan, 2000).

\section{Genetic deletion in mice and knockdown in zebrafish}

Mice with targeted deletions of Notch family genes exhibit diverse vascular defects, mostly affecting the process of vascular remodelling and arterial specification. Notch1, Dll4 and CSL mutations result in the most severe vascular disruptions. The Notch1 null mice die due to profound defects of angiogenic vascular remodelling, shown most obviously as a failure of proper formation of the major arteries. The mice form a primary vascular plexus, indicating that Notch signals are not required for vasculogenesis and early angiogenesis; however, this plexus fails to remodel into an organised network of larger and smaller vessels (Krebs et al, 2000). Mice lacking the Notch4 gene develop normally; however, Notch1/Notch4 double mutants show more severe vascular defects than mice lacking only Notch1, suggesting that Notch1 and Notch4 have partially redundant functions (Krebs et al, 2000). Proper vascular development requires appropriate Notch activity, as mice with an activated allele of Notch4 (Notch4/ int3) die in utero and display severe vascular anomalies, reminiscent of those seen in Notch1 null mice (Uyttendaele et al, 2001). Similarly, targeted endothelial expression of INT3 produces reversible arteriovenous defects in adult mice (Carlson et al, 2005). Jagged 1 deletion also results in lethality due to haemorrhage and failure in angiogenic remodelling (Xue et al, 1999). In support of its role as the primary endothelial cell Notch ligand, heterozygous deletion of Dll4 is lethal. Vascular defects in Dll4 mutants include arteriovenous malformations, a lack of vascular remodelling and collapse or incomplete formation of the major arteries (Duarte et al, 2004; Gale et al, 2004; Krebs et al, 2004). In rare-surviving Dll4 heterozygous mice, defects were also noted in sprouting angiogenesis in the neonatal retinal vessels (Lobov et al, 2007; Suchting et al, 2007); these defects are understood in terms of a failure for local cell fate decisions in the leading front of angiogenic vessels (see below). In zebrafish, Delta-Notch signalling has been shown to be important for the differentiation of arterial endothelial cells and proper formation of the major arteries (Lawson et al, 2001). In addition, Notch signalling is also required for proper control of angiogenic sprouting of, for example, the intersomitic vessels (Leslie et al, 2007; Siekmann and Lawson, 2007).

\section{Blockade of Dll4 in tumours}

As could be expected from its role in developmental angiogenesis, the Delta-Notch pathway is also important in tumour angiogenesis. 
Several studies have documented the expression of Notch components in tumour vessels, most notably Dll4. Studies of tumours in mice and humans have shown that Dll4 is strongly expressed in tumour blood vessels compared to adjacent normal vessels. For example, in human tumours, Dll4 expression was localised to the blood vessels of clear-cell renal tumours (Mailhos et al, 2001) and was at nine-fold higher levels than in normal kidney tissue (Patel et al, 2005). In keeping with the studies described above, expression of Dll4 in tumour vessels appears to be directly regulated by VEGF. For example, blockade of VEGF in tumour-bearing mice results in a rapid and profound reduction of Dll4 expression by the tumour blood vessels (Noguera-Troise et al, 2006). Conversely, the levels of Dll4 expression in tumours correlate with the levels of VEGF expression (Patel et al, 2006). Thus, the high levels of Dll4 expression on tumour vessels may be a result of relatively high levels of VEGF signalling in these vessels compared to most normal vessels.

The striking pattern of Dll4 expression in tumour vessels prompted several groups to target Dll4-Notch activity and provided insight into a role for Dll4-Notch in regulating VEGFinduced vascular sprouting. Local or systemic treatment of mice with Dll4-Notch inhibitors caused overgrowth of a non-functional tumour vasculature. However, strikingly, blockade of Dll4-Notch resulted in a growth inhibition in a variety of established human and rodent tumour models (Noguera-Troise et al, 2006; Ridgway et al, 2006; Scehnet et al, 2007). The reduced growth was associated with an increase in tumour vessel density and increased vessel sprouts with numerous interconnecting branches. Even though the tumours were smaller due to Dll4 blockade, tumours were more hypoxic, apparently containing non-functional vessels ('abnormalisation' - Thurston et al, 2007).

Other Notch ligands may also influence tumour angiogenesis; for example, one study suggests an angiogenic role for Jagged 1 in head and neck tumours (Zeng et al, 2005). A similar conclusion was reached in a study of a novel Notch inhibitor, the Notch1 decoy, which has the ability to block Notch signalling via several different Notch ligands, including both Dll4 and Jagged1. The Notch1 decoy was shown to block angiogenesis in both a VEGFdriven dermal angiogenesis model and a xenograft model of mammary tumour growth (Funahashi et al, 2008). It remains to be established which ligands are most critical for the antiangiogenic effect of the Notch1 decoy.

\section{INTERACTIONS OF VEGF AND DELTA-NOTCH PATHWAYS}

\section{Genetic evidence for interaction between VEGF and Delta-Notch pathways}

An initial appreciation of the relationship between the VEGF pathway and the Delta-Notch pathway came from studies of vascular development in zebrafish. Several studies have shown that loss of Notch signalling, for example with morpholino knockdown of Notch receptors or in mutants of the downstream gene gridlock, leads to defective development of the arterial vessels (Zhong et al, 2001). Subsequently, VEGF was shown to act upstream of Notch in determining arterial cell fate in vascular development (Lawson et al, 2002). By analogy to the studies of VEGF activity in cultured endothelial cells, it is possible that VEGF signalling leads to increased Dll4 and Notch expression, in turn leading to productive Notch signalling and thus arterial specification characterised by expression of a defined set of arterial genes. However, it is not clear whether a VEGF signal in isolation can induce de novo expression of Notch components (Lawson et al, 2002). More work will be needed to fully define the biochemical link between the VEGF pathway and the Delta-Notch pathway in this setting (Siekmann et al, 2008).

\section{VEGF increases Dll4 expression}

Studies in several systems, discussed here, establish that VEGF regulates the expression of Notch signalling components. Studies with cultured endothelial cells reveal several ways in which the VEGF and Dll4-Notch pathways interact. Importantly, VEGF increases Dll4 expression. Treatment of cultured endothelial cells with VEGF results in increased expression of Dll4 mRNA and protein (Patel et al, 2005; Hainaud et al, 2006; Ridgway et al, 2006). Vascular endothelial growth factor is a classic hypoxia response gene; thus the induction of Dll4 can occur indirectly in situations of hypoxia, via induction of VEGF. In addition, Dll4 expression by endothelial cells may be directly upregulated by hypoxia, possibly via Hif1 $-\alpha$ and hypoxia response elements in the Dll4 promoter (Patel et al, 2005; Diez et al, 2007).

The expression of Dll4 is also increased by VEGF in vivo. Dll4 is strongly expressed by endothelial cells of sprouting angiogenic vessels, which are commonly responding to VEGF signals. For example, in the developing murine retina, a VEGF gradient emanating from the avascular regions of the retina is essential for the sprouting angiogenesis. These growing vessels, and in particular the 'tip cells', are associated with strong expression of Dll4 (Hellstrom et al, 2007; Lobov et al, 2007). Blocking VEGF, by intravitreal injection of soluble VEGF receptors, results in decreased sprouting and decreased expression of Dll4 on the retinal vessels (Suchting et al, 2007). Conversely, intravitreal injection of VEGF protein increased expression of Dll4 in the retina (Lobov et al, 2007). Furthermore, in an oxygen-induced model of retinal angiogenesis (OIR), Dll4 expression is also increased on the newly forming retinal vessels (Lobov et al, 2007). Again, specific blockade of VEGF blocks the increased expression of Dll4 on the newly forming retinal vessels in the OIR model. It is noteworthy that blocking VEGF in this model does not reduce expression of Dll4 on arteries, indicating that other signals can also induce expression of Dll4.

Similar associations between VEGF signalling, growing vessels and Dll4 expression have been found in tumour vessels (Mailhos et al, 2001; Patel et al, 2005; Noguera-Troise et al, 2006). As described above, VEGF drives a key pathway for tumour angiogenesis in many models, and ongoing VEGF signalling is often required for sprouting angiogenesis in tumours. Increased expression of Dll4 in tumour vessels has been reported in several mouse tumour models (Mailhos et al, 2001; Noguera-Troise et al, 2006) and in human tumours (Patel et al, 2005). For example, Dll4 expression was found to be particularly strong on the growing front of vessels as tumours become vascularised (Noguera-Troise et al, 2006). Conversely, blockade of VEGF in tumours resulted in a rapid decrease of Dll4 expression in tumour vessels (NogueraTroise et al, 2006), showing that a significant portion of the Dll4 expression in the growing tumour vessels requires ongoing VEGF signalling. Thus, in tumours, VEGF can act to regulate the expression of Dll4, as schematised in Figure 1A. Other ligands for Notch, particularly Jagged1, have also recently been associated with angiogenic endothelial cells (Sainson et al, 2008). It will be very important to determine whether the different ligands provide the same Notch signalling activity in angiogenic endothelial cells.

\section{Dll4-Notch signalling affects expression of VEGF receptors}

Separate studies have suggested that Notch signalling can alter expression levels of all three VEGF receptors, schematised in Figure 1B. The best-characterised case is that of VEGF-R2, which is downregulated by either Notch or Heyl (a Notch target gene). Downregulation of VEGF-R2 has been observed following activation of Notch in cultured endothelial cells (Taylor et al, 2002). Reciprocally, increased VEGF-R2 levels were observed in vessels of Dll4 heterozygous mice or as a result of Dll4 blockade (Suchting et al, 2007). This important finding suggests that Notch can 
A VEGF regulates DII4-Notch

B

Notch regulates VEGF receptors
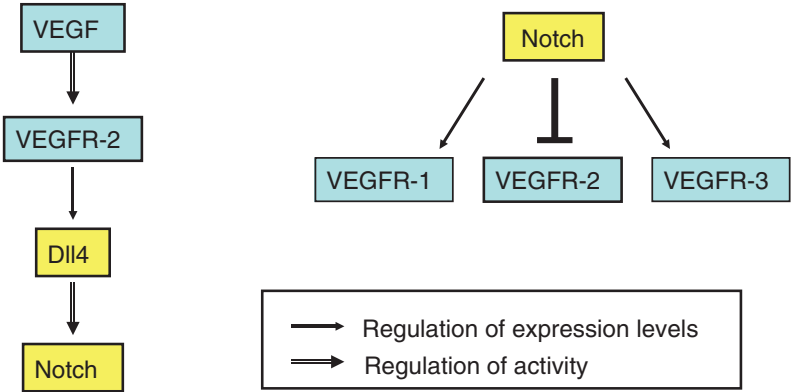

$\longrightarrow$ Regulation of expression levels $\longrightarrow$ Regulation of activity

Figure I Schematic diagram summarising the different ways in which the VEGF and Notch pathways interact. (A) VEGF stimulus, acting via VEGF$\mathrm{R} 2$, increases expression of DII4 on endothelial cells, which in turn activates Notch receptors on adjacent endothelial cells. (B) Activated Notch receptors on endothelial cells can in turn positively (VEGF-RI, VEGF-R3) or negatively (VEGF-R2) regulate the expression of VEGF receptors in those cells.

provide negative feedback to reduce the activity of the VEGF/ VEGF-R2 axis. In cultured endothelial cells, downregulation of VEGF-R2 may explain the reduced endothelial proliferation and migration seen as a result of Notch signal activation (Taylor et al, 2002). In vivo, downregulation of VEGF-R2 has been proposed as a mechanism to permit local differentiation of cells within a zone of VEGF-driven angiogenesis: high levels of VEGF induce Dll4 in an endothelial 'tip cell', which in turn provides a Notch signal and decreased VEGF-R2 in downstream 'stalk cells' (Roca and Adams, 2007). Through this pathway of local cell differentiation, the tip cells respond robustly to VEGF whereas the stalk cells respond in a more muted manner.

Two recent reports suggest that VEGF-R1 expression may be increased by Notch signalling. Reduced Notch activity, such as in Dll4 heterozygous mice, resulted in reduced VEGF-R1 expression, in addition to increased VEGF-R2 expression (Suchting et al, 2007). In addition, Dll4-Notch activity resulted in increased expression of both the signalling form of VEFR-1 and the soluble form of VEGF-R1 (sFlt1) in cultured endothelial cells (Harrington et al, 2008). VEGF-R1 is a less potent signalling receptor than VEGF-R2; thus it is not clear whether changing it's coexpression with VEGF-R2 would tend to increase, decrease or change the character of the signal.

A recent study describes the VEGF-R3 gene as a direct transcriptional target of the Notch signalling pathway, with NICD and the CSL transcription factors binding directly to sites within the human and murine VEGF-R3 promoters (Shawber et al, 2007). In addition to showing a dramatic increase in VEGF-R3 expression, Shawber et al (2007) also showed an alteration of endothelial VEGF responses as a result of Notch signal activation. Notch signalling increased the cellular responses to VEGF-C, presumably via upregulation of VEGF-R3, and decreased the response to VEGF-A, via downregulation of VEGF-R2. An analogous regulation of VEGF-R3 was also apparent in mouse embryos following increased or decreased Notch activity. Finally, although heterozygous Notch1 or VEGF-R1 mice are viable, doubly heterozygous Notch1-I+VEGFR1-I+ embryos often die due to vascular problems. The regulation of VEGF-R3 by Notch is likely complex, as not all vascular beds showed increased VEGF-R3 levels in response to Notch signalling. In zebrafish, Notch has been proposed to downregulate the VEGF-R3 homologue, zFlt4, during arterial specification and in the angiogenic tip cells of intersomitic vessels (Siekmann and Lawson, 2007). Thus, Notch may interact with the VEGF-R3 promoter in a context- and cell type-specific fashion leading to different outcomes in promoter regulation. In sum, these results suggest a complex interplay between Notch activity and expression of VEGF receptors, resulting in changes in how a particular endothelial cell will respond to VEGF signals.

\section{Combination blockade of VEGF and Dll4 is more effective in some tumours}

An exciting opportunity has arisen because recent studies have shown that blockade of Dll4 can have potent antitumour effects on tumours that are resistant to VEGF inhibition (Noguera-Troise et al, 2006; Ridgway et al, 2006). For example, the growth of a sarcoma model that is very resistant to VEGF blockade was strongly inhibited by blockade of Dll4-Notch (Noguera-Troise et al, 2006). In addition, the tumour vessels in this model showed dramatic disorganisation and dysfunction following Dll4-Notch blockade. Furthermore, simultaneously blocking both VEGF and Dll4 can have more potent effects in a variety of tumour models than blockade of either factor alone (Ridgway et al, 2006; I Noguera-Troise et al, unpublished results), including this sarcoma model. Previous studies have shown that individual blockade of VEGF and Dll4-Notch produces very different effects on the morphology and function of tumour vessels (above, also Thurston et al, 2007); however, to date, there is little information on the effects of blocking both pathways in combination.

Although these observations raise the possibility of using combination antiangiogenic therapies against the two pathways, much remains to be learned regarding how to best exploit this approach clinically. The factors produced by the tumour or expressed within the tumour vessels that confer sensitivity to Dll4Notch blockade are unknown. To be fair, it must be said that the corresponding factors that confer sensitivity to VEGF blockade are also largely unknown, despite intense investigation for a decade. A useful starting point may be to assume that high levels of Dll4Notch are in some way correlated with sensitivity to blockade. Thus, further information from clinical specimens on the levels of the various components of the VEGF and Delta-Notch pathways in different tumour types will be very useful.

Mechanistically, the observation of potent combination effects is difficult to fit into a simple model in which VEGF signalling induces Dll4-Notch activity in the vasculature, which subsequently helps to downregulate VEGF pathway activity in a neat feedback loop. It is clear that although this model may be fundamentally correct, other complexities are in play. For example, VEGF signalling induces a number of different downstream pathways and thus Dll4-Notch represents only one output. Conversely, Dll4 is also apparently upregulated by pathways other than VEGF, including in response to Notch signalling itself (Shawber et al, 2003; Ridgway et al, 2006). An interesting approach might be to consider whether Dll4 blockade is sensitising tumour vessels to VEGF blockade or vice versa. Alternatively, the combined blockade could be acting on separate subsets of tumour vessels within a heterogeneous network. Future studies to document the effect of combination blockade on the morphology and function of tumour vessels, combined with studies of the interaction of the pathways in cultured endothelial cells, are needed to shed further light on this issue.

\section{DISCUSSION AND FUTURE DIRECTIONS}

The formation of a hierarchical network of vessels requires the coordinated interplay of various signalling pathways. Even tumour vessels, which are structurally abnormal and poorly functional, can be rendered less efficient by interfering with vascular signalling pathways. As described in this review, two important signalling pathways with distinct roles in vascular development, namely the VEGF and Delta-Notch pathways, interact at several levels to help generate a hierarchical vessel network. Some of the interaction appears to be at the level of gene transcription; for example, the 


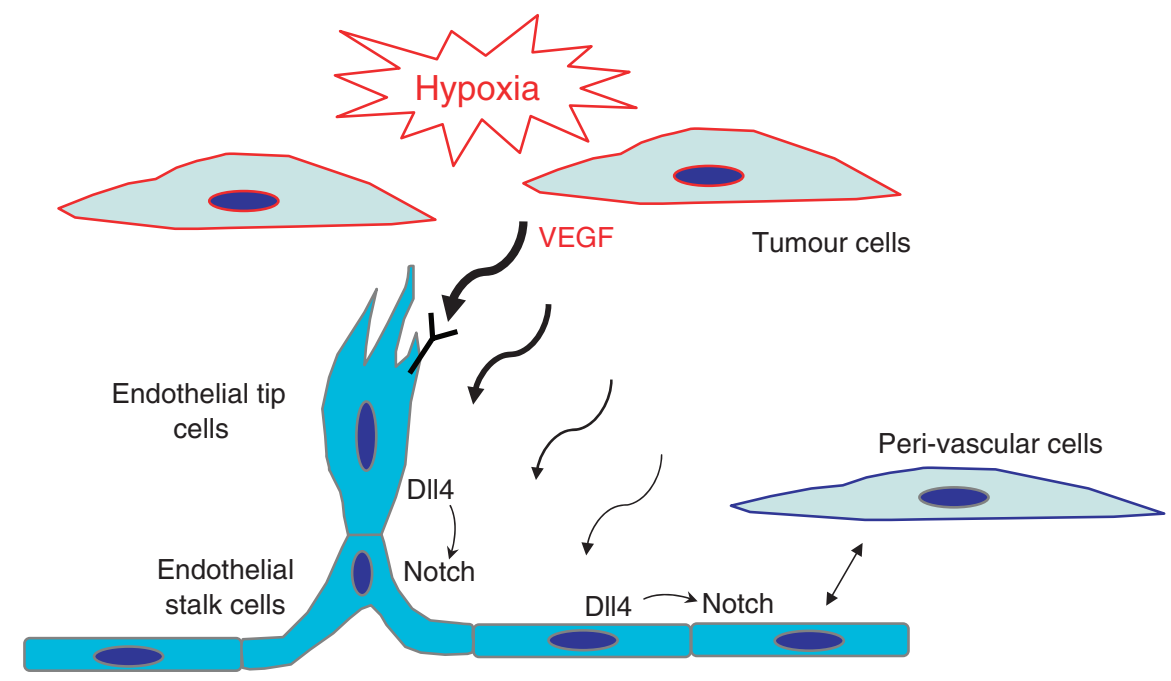

Figure 2 Comparison of the roles of VEGF and Delta-Notch pathways in angiogenesis and vascular development. Vascular endothelial growth factor from hypoxic tumour cells and surrounding tissue provides a signal to endothelial cells (via cell-restricted VEGF receptors) that calls for an increase in vascular function. The Delta-Notch pathway (particularly D\|l4) acts within the vasculature to help the endothelial cells respond appropriately to the activating VEGF signal. At least two settings in the developing vasculature apparently utilise VEGF as a driving signal and Delta-Notch to make cell fate decisions: the specialisation of tip cells and stalk cells within the growing front of angiogenic vessels and the specification of arterial cell fate.

activation of VEGF-R2 can lead to increased expression of Dll4. However, given the complexity of both the pathways, other levels of cross-control are likely and need to be explored.

Conceptually, the VEGF system provides a driving signal from surrounding tissues to endothelial cells (via cell-restricted VEGF receptors) that calls for an increase in vascular function. However, not all cells should respond equally to VEGF, and thus cell fate decisions are essential for generating an efficient response. Subsequently, the Delta-Notch pathway (particularly Dll4 and Notch1) acts among the endothelial cells to respond appropriately to the activating VEGF signal (Figure 2). In turn, an appropriate vascular response will provide increased vascular function and ultimately reduce the VEGF driving signal. Studies to date have indicated at least two settings in the developing vasculature in which VEGF drives a process and Delta-Notch helps to make cell fate decisions within that process; in particular, the specification of arterial cell fate and the specialisation of tip cells and stalk cells within the growing front of angiogenic vessels. It will be important to determine whether two different cell fate settings are applicable to the very chaotic process of tumour angiogenesis.

Further studies are needed to examine the interaction of the pathways in various tumour settings. We are just beginning to explore how we can take combinatorial advantage of the VEGF and Delta-Notch pathways for treating tumours, and a better conceptual framework within the context of tumour angiogenesis would help guide the experiments. Another issue will be to clarify some of the disparate results on the regulation of VEGF receptors by Delta-Notch activity: does VEGF receptor expression depend on endothelial cell phenotype, on the nature of Notch signal or on the presence of additional stimuli? Another important topic will be to determine how some of the key downstream Notch target genes (for example, ephrinB2) affect tumour vessels in the presence of high VEGF levels. Another potentially fruitful approach will be to examine whether other types of endothelial cell fate decisions in development are guided by Delta-Notch. We are at an exciting time for the convergence of basic vascular biology and tumour therapy.

\section{ACKNOWLEDGEMENTS}

We thank the following colleagues for helping to shape the ideas in this minireview: Irene Noguera-Troise, Ivan Lobov, Nicholas Gale, Calvin Lin, Stanley Wiegand and George Yancopoulos (Regeneron Pharmaceuticals), and Carrie Shawber (Columbia University).

\section{REFERENCES}

Carlson TR, Yan Y, Wu X, Lam MT, Tang GL, Beverly LJ, Messina LM, Capobianco AJ, Werb Z, Wang R (2005) Endothelial expression of constitutively active Notch4 elicits reversible arteriovenous malformations in adult mice. Proc Natl Acad Sci USA 102: 9884-9889

Diez H, Fischer A, Winkler A, Hu CJ, Hatzopoulos AK, Breier G, Gessler M (2007) Hypoxia-mediated activation of Dll4-Notch-Hey2 signaling in endothelial progenitor cells and adoption of arterial cell fate. Exp Cell Res 313: $1-9$

Duarte A, Hirashima M, Benedito R, Trindade A, Diniz P, Bekman E, Costa L, Henrique D, Rossant J (2004) Dosage-sensitive requirement for mouse Dll4 in artery development. Genes Dev 18: 2474-2478

Duda DG, Batchelor TT, Willett CG, Jain RK (2007) VEGF-targeted cancer therapy strategies: current progress, hurdles and future prospects. Trends Mol Med 13: 223-230

Ferrara N (1999) Role of vascular endothelial growth factor in the regulation of angiogenesis. Kidney Int 56: 794-814

Folkman J (2002) Role of angiogenesis in tumor growth and metastasis. Semin Oncol 29: 15-18

Funahashi Y, Hernandez S, Das I, Ahn A, Huang J, Vorontchinkhina M, Sharma A, Kanamaru E, Borisenko V, DeSilva DM, Suzuki A, Wang X, Shawber CJ, Kandel JJ, Yamashiro DJ, Kitajewski J (2008) A Notch1 ectodomain construct inhibits endothelial Notch signaling, tumor growth and angiogenesis. Cancer Res 68(12): 4727-4735 
Gale NW, Dominguez MG, Noguera I, Pan L, Hughes V, Valenzuela DM, Murphy AJ, Adams NC, Lin HC, Holash J, Thurston G, Yancopoulos GD (2004) Haploinsufficiency of delta-like 4 ligand results in embryonic lethality due to major defects in arterial and vascular development. Proc Natl Acad Sci USA 101: 15949-15954

Hainaud P, Contreres JO, Villemain A, Liu LX, Plouet J, Tobelem G, Dupuy E (2006) The role of the vascular endothelial growth factor-Delta-like 4 ligand/Notch4-ephrin B2 cascade in tumor vessel remodeling and endothelial cell functions. Cancer Res 66: 8501-8510

Harrington LS, Sainson RC, Williams CK, Taylor JM, Shi W, Li JL, Harris AL (2008) Regulation of multiple angiogenic pathways by Dll4 and Notch in human umbilical vein endothelial cells. Microvasc Res 75(2): 144-154

Hellstrom M, Phng LK, Hofmann JJ, Wallgard E, Coultas L, Lindblom P, Alva J, Nilsson AK, Karlsson L, Gaiano N, Yoon K, Rossant J, IruelaArispe ML, Kalen M, Gerhardt H, Betsholtz C (2007) Dll4 signalling through Notch1 regulates formation of tip cells during angiogenesis. Nature 445: 776-780

Krebs LT, Shutter JR, Tanigaki K, Honjo T, Stark KL, Gridley T (2004) Haploinsufficient lethality and formation of arteriovenous malformations in Notch pathway mutants. Genes Dev 18: 2469-2473

Krebs LT, Xue Y, Norton CR, Shutter JR, Maguire M, Sundberg JP, Gallahan D, Closson V, Kitajewski J, Callahan R, Smith GH, Stark KL, Gridley T (2000) Notch signaling is essential for vascular morphogenesis in mice. Genes Dev 14: 1343 - 1352

Lawson ND, Scheer N, Pham VN, Kim CH, Chitnis AB, Campos-Ortega JA, Weinstein BM (2001) Notch signaling is required for arterial-venous differentiation during embryonic vascular development. Development 128: $3675-3683$

Lawson ND, Vogel AM, Weinstein BM (2002) sonic hedgehog and vascular endothelial growth factor act upstream of the Notch pathway during arterial endothelial differentiation. Dev Cell 3: 127-136

Leslie JD, Ariza-McNaughton L, Bermange AL, McAdow R, Johnson SL, Lewis J (2007) Endothelial signalling by the Notch ligand Delta-like 4 restricts angiogenesis. Development 134: 839-844

Lobov IB, Renard RA, Papadopoulos N, Gale NW, Thurston G, Yancopoulos GD, Wiegand SJ (2007) Delta-like ligand 4 (Dll4) is induced by VEGF as a negative regulator of angiogenic sprouting. Proc Natl Acad Sci USA 104: 3219-3224

Mailhos C, Modlich U, Lewis J, Harris A, Bicknell R, Ish-Horowicz D (2001) Delta4, an endothelial specific notch ligand expressed at sites of physiological and tumor angiogenesis. Differentiation 69: 135-144

Mumm JS, Kopan R (2000) Notch signaling: from the outside in. Dev Biol 228: $151-165$

Nasevicius A, Larson J, Ekker SC (2000) Distinct requirements for zebrafish angiogenesis revealed by a VEGF-A morphant. Yeast 17: 294-301

Noguera-Troise I, Daly C, Papadopoulos NJ, Coetzee S, Boland P, Gale NW, Lin HC, Yancopoulos GD, Thurston G (2006) Blockade of Dll4 inhibits tumour growth by promoting non-productive angiogenesis. Nature 444: $1032-1037$

Patel NS, Dobbie MS, Rochester M, Steers G, Poulsom R, Le Monnier K, Cranston DW, Li JL, Harris AL (2006) Up-regulation of endothelial deltalike 4 expression correlates with vessel maturation in bladder cancer. Clin Cancer Res 12: $4836-4844$

Patel NS, Li JL, Generali D, Poulsom R, Cranston DW, Harris AL (2005) Up-regulation of delta-like 4 ligand in human tumor vasculature and the role of basal expression in endothelial cell function. Cancer Res 65: $8690-8697$

Ridgway J, Zhang G, Wu Y, Stawicki S, Liang WC, Chanthery Y, Kowalski J, Watts RJ, Callahan C, Kasman I, Singh M, Chien M, Tan C, Hongo JA, de Sauvage F, Plowman G, Yan M (2006) Inhibition of Dll4 signalling inhibits tumour growth by deregulating angiogenesis. Nature 444: $1083-1087$

Roca C, Adams RH (2007) Regulation of vascular morphogenesis by Notch signaling. Genes Dev 21: 2511-2524

Sainson RC, Johnston DA, Chu HC, Holderfield MT, Nakatsu MN, Crampton SP, Davis J, Conn E, Hughes CC (2008) TNF primes endothelial cells for angiogenic sprouting by inducing a tip cell phenotype. Blood 111(10): 4997-5007

Scehnet JS, Jiang W, Kumar SR, Krasnoperov V, Trindade A, Benedito R, Djokovic D, Borges C, Ley EJ, Duarte A, Gill PS (2007) Inhibition of Dll4mediated signaling induces proliferation of immature vessels and results in poor tissue perfusion. Blood 109: 4753-4760

Shawber CJ, Das I, Francisco E, Kitajewski J (2003) Notch signaling in primary endothelial cells. Ann NY Acad Sci 995: 162-170

Shawber CJ, Funahashi Y, Francisco E, Vorontchikhina M, Kitamura Y, Stowell SA, Borisenko V, Feirt N, Podgrabinska S, Shiraishi K, Chawengsaksophak K, Rossant J, Accili D, Skobe M, Kitajewski J (2007) Notch alters VEGF responsiveness in human and murine endothelial cells by direct regulation of VEGFR-3 expression. J Clin Invest 117: 3369-3382

Siekmann AF, Covassin L, Lawson ND (2008) Modulation of VEGF signalling output by the Notch pathway. Bioessays 30: $303-313$

Siekmann AF, Lawson ND (2007) Notch signalling limits angiogenic cell behaviour in developing zebrafish arteries. Nature 445: 781-784

Suchting S, Freitas C, le Noble F, Benedito R, Breant C, Duarte A, Eichmann A (2007) The Notch ligand Delta-like 4 negatively regulates endothelial tip cell formation and vessel branching. Proc Natl Acad Sci USA 104: $3225-3230$

Tammela T, Enholm B, Alitalo K, Paavonen K (2005) The biology of vascular endothelial growth factors. Cardiovasc Res 65: 550-563

Taylor KL, Henderson AM, Hughes CC (2002) Notch activation during endothelial cell network formation in vitro targets the basic HLH transcription factor HESR-1 and downregulates VEGFR-2/KDR expression. Microvasc Res 64: $372-383$

Thurston G, Noguera-Troise I, Yancopoulos GD (2007) The Delta paradox: DLL4 blockade leads to more tumour vessels but less tumour growth. Nat Rev Cancer 7: 327-331

Uyttendaele H, Ho J, Rossant J, Kitajewski J (2001) Vascular patterning defects associated with expression of activated Notch4 in embryonic endothelium. Proc Natl Acad Sci USA 98: 5643-5648

Xue Y, Gao X, Lindsell CE, Norton CR, Chang B, Hicks C, Gendron-Maguire M, Rand EB, Weinmaster G, Gridley T (1999) Embryonic lethality and vascular defects in mice lacking the Notch ligand Jagged1. Hum Mol Genet 8: 723-730

Zeng Q, Li S, Chepeha DB, Giordano TJ, Li J, Zhang H, Polverini PJ, Nor J, Kitajewski J, Wang CY (2005) Crosstalk between tumor and endothelial cells promotes tumor angiogenesis by MAPK activation of Notch signaling. Cancer Cell 8: 13-23

Zhong TP, Childs S, Leu JP, Fishman MC (2001) Gridlock signalling pathway fashions the first embryonic artery. Nature 414: 216-220 